\title{
A Dynamic Particle Swarm Optimisation and Fuzzy Clustering Means Algorithm for Segmentation of Multimodal Brain Magnetic Resonance Image Data
}

\author{
Kies Karima and Benamrane Nacera \\ Department of Computer Science, Université des Sciences et de la Technologie d'Oran "Mohamed \\ Boudiaf", Algeria
}

\begin{abstract}
Fuzzy Clustering Means (FCM) algorithm is a widely used clustering method in image segmentation, but it often falls into local minimum and is quite sensitive to initial values which are random in most cases. In this work, we consider the extension to FCM to multimodal data improved by a Dynamic Particle Swarm Optimization (DPSO) algorithm which by construction incorporates local and global optimization capabilities. Image segmentation of three-variate MRI brain data is achieved using FCM-3 and DPSOFCM-3 where the three modalities T1-weighted, T2-weighted and Proton Density (PD), are treated at once (the suffix -3 is added to distinguish our three-variate method from mono-variate methods usually using TIweighted modality). FCM-3 and DPSOFCM-3 were evaluated on several Magnetic Resonance (MR) brain images corrupted by different levels of noise and intensity non-uniformity. By means of various performance criteria, our results show that the proposed method substantially improves segmentation results. For noisiest and most no-uniform images, the performance improved as much as $9 \%$ with respect to other methods.
\end{abstract}

Keywords: Fuzzy c-mean, particle swarm optimization, brain Magnetic Resonance Images segmentation.

Received December 24, 2019; accepted March 10, 2020

https://doi.org/10.34028/iajit/17/6/16

\section{Introduction}

Image segmentation is a crucial task in image processing and has a wide range of applications [27]. It is the process of partitioning an image into regions corresponding to different objects or classes according to predefined homogeneity criteria. A great number of methods for image segmentation are present in the literature. Image segmentation is essentially a clustering problem where the features embodying in each data element (pixel or voxel in the simplest definition) correspond to a pattern, and each image region corresponds to a cluster [10].

In this work we consider the segmentation of Brain Magnetic Resonance Images (MRI) into Grey Matter (GM), White Matter (WM) and Cerebrospinal Fuid (CSF). An overall review of state of the art methods, potential issues and challenges can be found here [7, 12].

In this paper we present the background and related work in section 2. And then, we introduce the standard Fuzzy Clustering Means (FCM) algorithm in section 3. In section 4, the standard Particle Swarm Optimization (PSO) algorithm is reviewed. We describe our proposed method on section 5. In section 6 we present our results and discuss performance as compared to other work. At last, conclusions are drawn in section 7 .

\section{Background and Related Work}

Introduced by Dunn [8] and generalised by Bezdek [4], the standard FCM algorithm is among the most widely used fuzzy clustering method for images segmentation. Most authors have used FCM for Brain image segmentation using a single modality usually T1weighted [1]. Other authors have used more than one modality (T2- and PD weighted) [11, 19]. But, as far as we know, none have used the three modalities at once in order to characterise the feature for each data element. The application of standard FCM algorithm to MRI brain image segmentation has limited performance since outcome greatly depends on the initial cluster centres. So, the algorithm falls quite often into locally optimal solutions and misses global ones. Another drawback of FCM is its high sensitivity to MR image artefacts, such as noise, and intensity inhomogeneity. A nice review for general applications and improvement of FCM Clustering Algorithm can be found here [22], and a good review of specific FCM algorithms for brain MR Images segmentation can be found here [7]. 
Many bio-inspired techniques such as Genetic Algorithm (GA), Simulated Annealing (SA), Ant Colony Optimization (ACO) and also PSO, were proposed in addition to $\mathrm{FCM}$ in order to reduce its inadequacies [9].

Very recently, other very promising heuristic and metaheuristic approaches have been used to solve various optimization problems and may open new perspectives and further improve image segmentation problems. Among these approaches, we have: humpback whale optimization [18], grey wolf optimizer [3], most valuable player algorithm [14] and sea lion optimization algorithm [17].

Because of its simplicity and efficiency, PSO, has been successfully used in conjunction with FCM on a wide variety of clustering problems [21, 25]. In most cases, each particle is considered as a candidate cluster centre and particles move in the solution space to search optimal cluster centres.

One way of combining FCM and PSO for image segmentation is to use FCM algorithm to find cluster centres that maximizes a given similarity function or minimizes a given dissimilarity function. Then, PSO is applied for labelling each pixel to a cluster. Another way is to start with PSO to determine initial clusters and then use FCM for labelling after defuzzification.

In this article, we will introduce the standard FCM algorithm in section 2. In section 3, the standard PSO algorithm is reviewed. We describe our proposed method on section 4 . In section 5 we present our results and discuss performance as compared to other work. Conclusions are drawn is the last section.

\section{Fuzzy C-Means Clustering (FCM)}

FCM is a general purpose data clustering algorithm in which data points are assigned fuzzy membership to a finite number of clusters. This algorithm was proposed as an improvement to the well-known k-means clustering algorithm where data points are assigned a unique (crisp) membership. The FCM algorithm is an iterative algorithm that produces an optimal partition of input data set $\Omega$ of size $N$ into a cluster set $\mathrm{Y}$ of size $c$. Each data point $x_{k}$ from $\Omega$ is assigned a membership vector of size $c, u_{j k}$ where the index $j$ runs from 1 to $c$, and index $k$ runs from 1 to $N$. We shall use $U$ as the set of all $u_{j k}$. The standard FCM objective function is:

$$
J_{m}(U, Y)=\sum_{k=1}^{N} \sum_{j=1}^{c}\left(u_{j k}\right)^{m}\left\|x_{k}-y_{j}\right\|^{2}
$$

Where $m \geq 1$ is a control parameter of fuzziness, $y_{j}$ the cluster centroids and $\|$ is the standard Euclidean distance. The clustering problem can be defined as the minimization of $J_{m}$ under the following probabilistic constraint:

$$
\sum_{j=1}^{c} u_{j k}=1
$$

As proposed by Bezdek [4], the FCM algorithm consists in the iteration of the following formulas:

For $j \in[1, \mathrm{c}]$, the centroids are given by:

$$
y_{j}=\frac{\sum_{k=1}^{N}\left(u_{j k}\right)^{m} x_{k}}{\sum_{k=1}^{N}\left(u_{j k}\right)^{m}}
$$

And for $j \in[1, \mathrm{c}], k \in[1, N]$, the membership factors are given by:

$$
u_{j k}=\left(\sum_{l=1}^{c}\left(\frac{\left\|x_{k}-y_{j}\right\|^{2}}{\left\|x_{k}-y_{l}\right\|^{2}}\right)^{\frac{1}{m-1}}\right)^{-1}
$$

After setting the number of cluster $c$ and the fuzziness factor $m$, there are essentially two variant for the iterative process. In the first variant, random centroids $y_{j}$ are chosen and then Equation (4) is used to calculate the membership factors $u_{j k}$. In the second variant, random membership factors $u_{j k}$ are chosen and then Equation (3) is used instead. The process is repeated until some stopping criteria are reached.

The $y_{j}$ and $u_{j k}$ obtained at the end of the iterative process represent the solution of the clustering problem. As mentioned earlier, we will refer to $Y$ as the set of all elements $\left(y_{j}\right)$ and to $U$ as the set of all elements $\left(u_{j k}\right)$. The final labelling is usually done using the partition matrix $U$ according to Equation (5)

$$
c_{i}=\arg _{k}\left\{\max \left(u_{k i}\right)\right\} ; i \in[1, N] ; k \in[1, c]
$$

\section{Particle Swarm Optimisation (PSO)}

The algorithm of particle swarm optimization is an evolutionary computational model introduced by Kennedy and Eberhart [13]. It gained interest very quickly from the scientific community involved in many fields where optimization algorithms are needed. PSO optimizes a problem by having a set (called swarm or population) of $n$ candidate solutions $x_{p}$ called particles which move around in the search-space according to simple mathematical formulae. The movements of the particles are guided by the best found positions in the search-space which are then updated whenever better positions are found.

The PSO algorithm requires the following variables:

1. $\mathrm{X}=$ Set $\left\{x_{p} / p \in[1, n]\right\}$, of current positions of the particles

2. $\mathrm{V}=$ Set $\left\{v_{p} / p \in[1, n]\right\}$ of current velocities for each particle.

3. $\mathrm{B}=$ Set $\left\{b_{p} / p \in[1, n]\right\}$ of personal best positions for each particle. 
4. $G$, the global best position for the whole set of $n$ particles.

At each step $t$, these variables are updated using the following key equations:

$$
\begin{gathered}
V(t+1)=\omega V(t)+c_{1} r_{1}(t)(B(t)-X(t)) \\
+c_{2} r_{2}(t)(G(t)-X(t)) \\
X(t+1)=X(t)+V(t+1)
\end{gathered}
$$

Where, $\omega$ is the inertia weight which controls the impact of previous velocity of particle on its current one, $c_{1}$ and $c_{2}$ are positive constants, called acceleration coefficients which control the influence of personal best performance and global best performance on the search process, $r_{1}$ and $r_{2}$ are random values in range [0, $1]$.

The performance of each particle is measured using a predefined fitness function $f$. The personal best performance and global best performance are updated as follow:

And:

$$
\begin{gathered}
b_{p}(t+1)=\left\{\begin{array}{c}
b_{p}(t) \text { if } f\left(x_{p}(t+1)\right) \geq f\left(b_{p}(t)\right) \\
x_{p}(t+1) \text { if } f\left(x_{p}(t+1)\right)<f\left(b_{p}(t)\right)
\end{array}\right. \\
G(t) \in\left\{b_{p}(t)\right\}=\min \left\{f\left(b_{p}(t)\right)\right\}
\end{gathered}
$$

Where $p \in[1, n]$.

The iterative process starts by choosing random $\mathrm{X}$ and $\mathrm{V}$, these quantities evolve according to formulae Equations (5), (6), and (7). The process stops when the global fitness $G$ converges, i.e., $|G(t+1)-G(t)|<\varepsilon$, where $\varepsilon$ is the desired precision or when some given maximum of iteration iter $_{\max }$ is exceeded. The final value obtained for $\mathrm{G}$ represents the solution for the optimisation.

In the evaluation of population based iterative optimization algorithms, local optima avoidance is a primary objective. PSO uses the contribution of global best for updating velocity of particles and influence their position which leads to a rapid convergence. But, it is highly vulnerable of getting trapped in local optima, specifically in complex multimodal problems which results in premature convergence. Many different PSO have been developed with the goal to improve the performance. In this paper, we will use a variant of PSO named Dynamic-PSO (DPSO) [24], which overcomes the problem of getting trapped in local optima with little effect on the fast convergence characteristic of PSO. The DPSO approach dynamically adapts PSO parameters such as $\omega$ the inertia weight as well as and the acceleration coefficients $c_{1}$ and $c_{2}$ as shown, respectively, on Equations (9), (10) and (11).

$$
\begin{gathered}
\omega=\left\{\begin{array}{c}
\omega_{i}-\frac{\left(\omega_{f}-\omega_{i}\right) \times\left(f_{p}-f_{\min }\right)}{\left(f_{\text {wog }}-f_{\min }\right)} ; f_{p} \leq f_{\text {wrg }} \\
\omega_{f} ; f_{p}>f_{\text {wog }}
\end{array}\right. \\
c_{1}=c_{i 1}+\frac{\left(c_{f 1}-c_{i 1}\right)}{i t e r_{\max }} \times \text { iter } \\
c_{2}=c_{i 2}+\frac{\left(c_{f 2}-c_{i 2}\right)}{i \text { iter } r_{\max }} \times \text { iter }
\end{gathered}
$$

Where $\omega_{\mathrm{f}}$ is the maximum inertia weight and $\omega_{\mathrm{i}}$ is the smallest inertia weight. And, $f_{p}$ is current fitness of particle $p$ and $f_{\text {avg }}$ is the current average fitness of all particles and $f_{\min }$ is minimum fitness of all particles. Similarly; $c_{\mathrm{i} 1}, c_{\mathrm{i} 2}, c_{\mathrm{f} 1}$ and $c_{\mathrm{f} 2}$ are respectively the initial and final values for $c_{1}$ and $c_{2}$.

The fitness for each particle is given by:

$$
f=\sum_{k=1}^{N} \sum_{j=1}^{c}\left\|x_{k}-y_{j}\right\|^{2}
$$

\section{The Proposed Method}

It is well known that FCM algorithm has some limitations that require the number of cluster and starting set of cluster centroids. And therefore, there have been several suggested algorithms that combine FCM and PSO to perform image segmentation. Generally, PSO algorithm is used for the purpose of initialisation of cluster centroids or membership factors [16]. In some other methods, FCM may be used as fitness of PSO or FCM may be used to trigger PSO. Also, one may also combine sequentially FCM-PSOFCM.

In this paper, we propose a method combining Dynamic PSO and FCM for the segmentation of white matter, grey matter and cerebrospinal fluid using MRI images of the brain. In particular, three volumetric data sets representing T1-weighted, T2-weighted, and PD weighted MRI data taken from Brainweb database [5]. In previous work, these three volumetric data have been segmented separately. In this work, the fusion of the three data sets produces a three-variate volume, so that the feature space is three dimensional where each triplet of voxel intensity is represented. This method can be extended to more than three-variate data. Our algorithm flow chart is shown on Figure 1. 


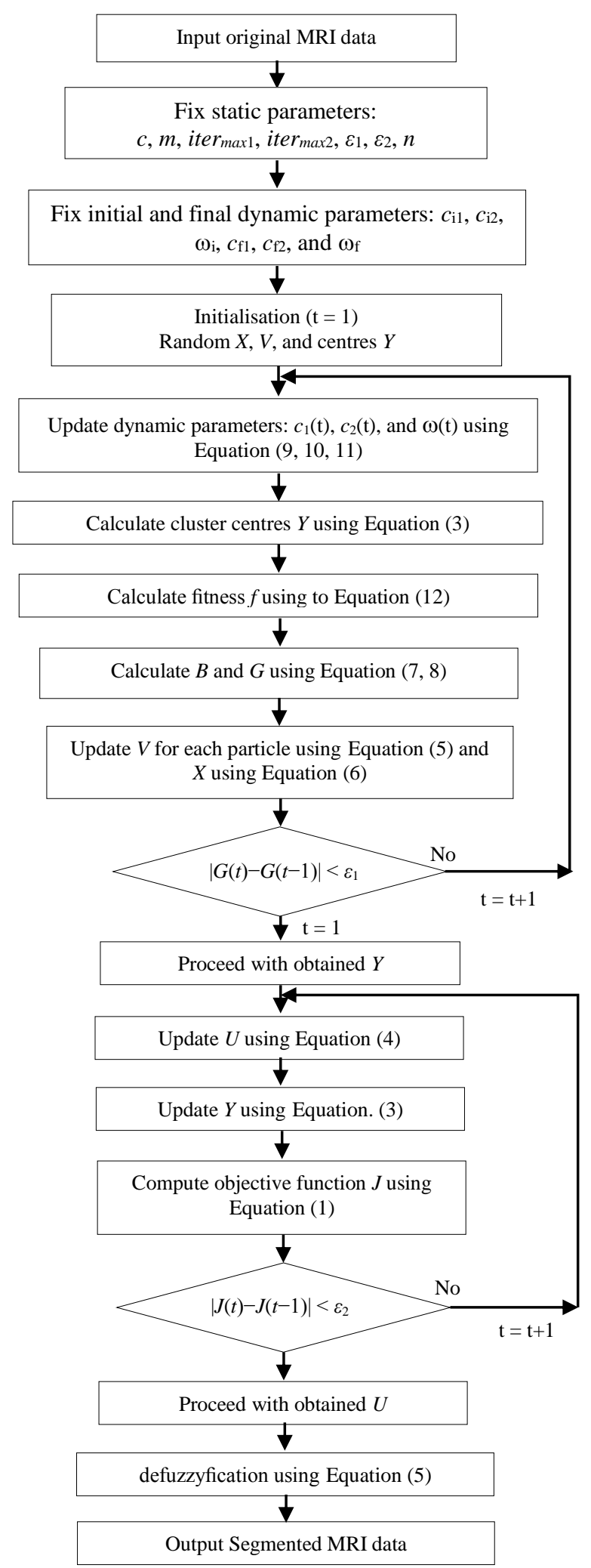

Figure 1. Algorithm flowchart.

To evaluate the quality of a partition provided our algorithm; we use the well-known measure which is the Dice index measure. There are many other measures used in the Literature, but the Dice index seems to be more intuitive because it can be seen as the percentage of overlap between the experimental set and the ground truth set. It is a number between 0 and 1. The Dice index or overlap ratio (DC) is defined as:

$$
D C=2 \times \frac{\left|S_{1} \cap S_{2}\right|}{\left|S_{1}\right|+\left|S_{2}\right|} \times 100
$$

This proposed algorithm will be named DPSOFCM3 where we added 3 to emphasize the use a three-variate data space at once. For an $\mathrm{N}$-variate data space, it would be named DFCMPSO-N.

\section{Results and Discussion}

In this section, we display results of the proposed algorithm DPSOFCM-3 as compared to FCM-3 and some other algorithms on the same set of simulated MRI brain data downloaded from Brainweb database [6].

The testing data $(181 \times 217 \times 181$ voxels $)$ are from three MRI modalities ( $\mathrm{T}_{1}, \mathrm{~T}_{2}$ and $\left.\mathrm{PD}\right)$, corrupted by six levels $(0 \%, 1 \%, 3 \%, 5 \%, 7 \%, 9 \%)$ of white Gaussian Noise (GN) and three intensity $(0 \%, 20 \%, 40 \%)$ Radio Frequency non-uniformity (RF).

Our algorithms were implemented in MATLAB V 2019a. The study was performed using the following parameters (see end of section 7):

- number of cluster $c=4$, BackGround (BG), GM, WM, CSF,

- $\mathrm{m}=2$ ( we checked that $\mathrm{m} \geq 2$ is enough to ensure less than $1 \%$ accuracy)

- iter $_{\max 1}=$ iter $_{\max 2}=100$

- $\varepsilon_{1}=\varepsilon_{2}=10^{-6}$,

- $\mathrm{c}_{\mathrm{i} 1}=\mathrm{c}_{\mathrm{f} 2}=2.5$ and $\mathrm{c}_{\mathrm{i} 2}=\mathrm{c}_{\mathrm{f} 1}=0.5$

- $\omega_{\mathrm{i}}=0.4$ and $\omega_{\mathrm{f}}=0.9$ and

- $n=20$.

Firstly, we present some qualitative FCM-3 segmentation results for slice \#77 (S77), which is a set of the three modalities $\mathrm{T}_{1}, \mathrm{~T}_{2}$, and PD images of $181 \times 217$ pixels. On Figure 2, we display one element of our work basis (which counts 181 elements). Namely, the three modalities of the original data and the segmentation into GM, WM and CSF ground truth.

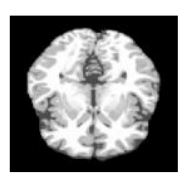

a) $\mathrm{T}_{1}$.

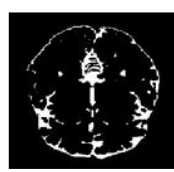

d) CSF.

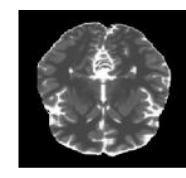

b) $\mathrm{T}_{2}$.

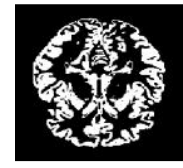

e) GM.

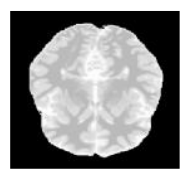

c) $\mathrm{PD}$.

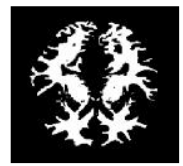

f) WM.
Figure 2. Skull stripped original MRI (S77, 0\%GN, 0\% RF) ground truth. 


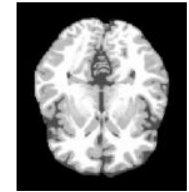

a) $T_{1}$

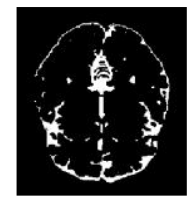

d) CSF.

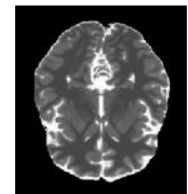

b) $\mathrm{T}_{2}$

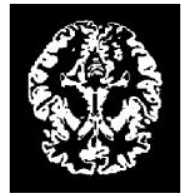

e) GM.

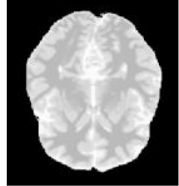

c) PD

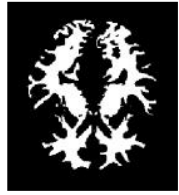

f) WM.
Figure 3. Skull stripped original MRI (S77, no noise, no RF nonuniformity) FCM-3 segmentation.

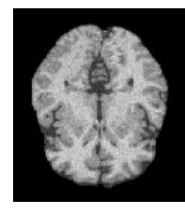

a) $T_{1}$.

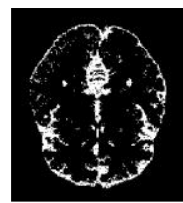

d) CSF.

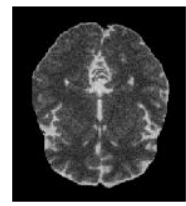

b) $\mathrm{T} 2$.

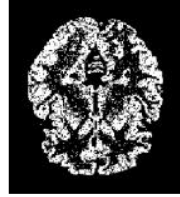

e) GM.

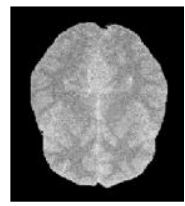

c) PD.

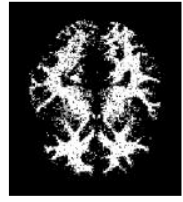

f) WM.
Figure 4. Skull stripped original MRI (S77, 9\% noise, 20\% RF nouniformity) FCM-3 segmentation.

The FCM-3 segmentation result for S77 with no Gaussian noise and no RF is shown on Figure 3. We can see clearly that it agrees quite well with ground truth. This confirms the well-known fact that FCM converges quite well in images with no noise.

On Figure 4, the FCM-3 segmentation drawback is clearly seen on noisy data.

This drawback persists even when using FCM-3, even though FCM-3 performs better than the standard FCM which uses one modality. A noticeable improvement is achieved by our DPSOFCM-3 algorithm as can be seen on Figure 5.

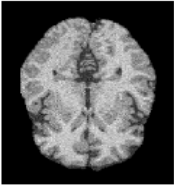

a) $\mathrm{T}_{1}$

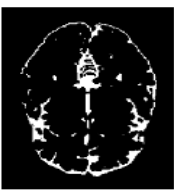

d) CSF.

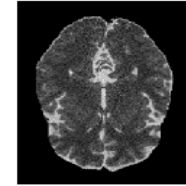

b) $\mathrm{T} 2$.

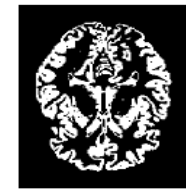

e) GM.

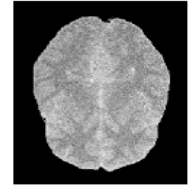

c) PD.

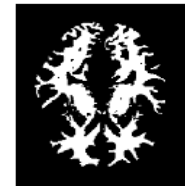

f) WM.
Figure 5. Skull stripped original MRI (S77, 9\% noise, 20\% RF nouniformity) DPSOFCM-3 segmentation.
Table 1. WM Dice (DC) Results for FCM-3 and DPSOFCM-3.

\begin{tabular}{|c|c|c|c|}
\hline \multirow{2}{*}{ DC (\%) } & \multicolumn{3}{|c|}{ RF (\%) } \\
\cline { 2 - 4 } Noise (\%) & \multicolumn{3}{|c|}{ Best T1:[21,24,28,29] } \\
\hline $\mathbf{0}$ & 98.00 & 97.00 & $\mathbf{4 0}$ \\
\hline $\mathbf{1}$ & 97.00 & 97.00 & 95.00 \\
\hline $\mathbf{3}$ & 95.00 & 96.00 & 94.00 \\
\hline $\mathbf{5}$ & 95.00 & 94.00 & 92.00 \\
\hline $\mathbf{7}$ & 93.00 & 92.00 & 90.00 \\
\hline $\mathbf{9}$ & 91.00 & 87.00 & 87.00 \\
\hline & & $\mathbf{3 C M - 3}$ \\
\hline $\mathbf{0}$ & 98.20 & 97.83 & 97.04 \\
\hline $\mathbf{1}$ & 97.96 & 97.63 & 96.88 \\
\hline $\mathbf{3}$ & 96.49 & 96.04 & 95.80 \\
\hline $\mathbf{5}$ & 94.07 & 93.94 & 93.40 \\
\hline $\mathbf{7}$ & 91.21 & 90.95 & 91.03 \\
\hline $\mathbf{9}$ & 87.07 & 87.74 & 87.32 \\
\hline & 98.45 & 98.08 & 97.29 \\
\hline $\mathbf{0}$ & 98.46 & 98.13 & 97.38 \\
\hline $\mathbf{1}$ & 97.49 & 97.04 & 96.80 \\
\hline $\mathbf{3}$ & 95.57 & 95.44 & 94.90 \\
\hline $\mathbf{5}$ & 93.21 & 93.05 & 93.03 \\
\hline $\mathbf{7}$ & 92.87 & 92.84 & 92.22 \\
\hline $\mathbf{9}$ & \multicolumn{3}{|c|}{ DPSOFCM-3 } \\
\hline
\end{tabular}

In the rest of this section, we will present tables with quantitative results in terms of the $\mathrm{DC}$ as given in Equation (9). We will show the DC for WM, GM, and CSF. Since the original images where already skull striped, the background DC is almost 1.00 .

On Table1, we compare our results for WM dice with best known results for T1-weighted modality. We can see that overall, FCM-3 is already as good without hybridation.

Table 2. GM Dice (DC) results for FCM-3 and DPSOFCM-3.

\begin{tabular}{|c|c|c|c|}
\hline \multirow{2}{*}{ DC (\%) } & \multicolumn{3}{|c|}{ RF (\%) } \\
\cline { 2 - 4 } Noise (\%) & $\mathbf{3}$ & $\mathbf{2 0}$ & $\mathbf{4 0}$ \\
\hline $\mathbf{0}$ & 97.27 & 96.87 & 95.96 \\
\hline $\mathbf{1}$ & 97.08 & 96.68 & 95.80 \\
\hline $\mathbf{3}$ & 95.75 & 95.09 & 94.70 \\
\hline $\mathbf{5}$ & 92.90 & 92.90 & 92.28 \\
\hline $\mathbf{7}$ & 90.01 & 89.77 & 89.83 \\
\hline $\mathbf{9}$ & 85.67 & 86.23 & 85.81 \\
\hline & \multicolumn{3}{|c|}{ DPSOFCM-3 } \\
\hline $\mathbf{0}$ & 97.52 & 97.12 & 96.21 \\
\hline $\mathbf{1}$ & 97.58 & 97.18 & 96.30 \\
\hline $\mathbf{3}$ & 96.75 & 96.09 & 95.70 \\
\hline $\mathbf{5}$ & 95.40 & 94.40 & 93.78 \\
\hline $\mathbf{7}$ & 94.01 & 93.77 & 92.83 \\
\hline $\mathbf{9}$ & 93.17 & 92.73 & 92.31 \\
\hline
\end{tabular}


Table 3. CSF Dice (DC) results for FCM-3 and DPSOFCM-3.

\begin{tabular}{|c|c|c|c|}
\hline \multirow{2}{*}{ DC (\%) } & \multicolumn{3}{|c|}{ RF (\%) } \\
\cline { 2 - 4 } & \multicolumn{3}{|c|}{ FCM-3 } \\
\hline & 96.49 & 96.24 & $\mathbf{4 0}$ \\
\hline $\mathbf{0}$ & 96.58 & 96.24 & 95.66 \\
\hline $\mathbf{1}$ & 96.50 & 95.75 & 95.59 \\
\hline $\mathbf{3}$ & 94.89 & 95.19 & 95.21 \\
\hline $\mathbf{5}$ & 94.51 & 94.50 & 94.31 \\
\hline $\mathbf{7}$ & 93.21 & 92.66 & 92.92 \\
\hline $\mathbf{9}$ & \multicolumn{3}{|c|}{ DPSOFCM-3 } \\
\hline & 96.74 & 96.49 & 95.91 \\
\hline $\mathbf{0}$ & 97.08 & 96.74 & 96.04 \\
\hline $\mathbf{1}$ & 97.50 & 96.75 & 96.21 \\
\hline $\mathbf{3}$ & 96.39 & 96.69 & 96.12 \\
\hline $\mathbf{5}$ & 96.51 & 96.50 & 96.13 \\
\hline $\mathbf{7}$ & 96.24 & 96.16 & 96.02 \\
\hline $\mathbf{9}$ & \multicolumn{3}{|c|}{} \\
\hline
\end{tabular}

As can be seen quantitatively, the new algorithm improves substantially the quality of the segmentation into the four principal classes. We have compared our results with best results found in the literature where the same database has been used and also where the similar comparison criteria have been used [20, 23, 28, 29] (in these references, authors have also compared their results with others that we have not included here to avoid repetition).

We can read from the numbers on Tables 1, 2, and 3 , that for $0 \%$ noise, and as expected, the improvement is overall less than 1\%. For largest Noise value, DPSOFCM-3 performs better than FCM-3: 4\% for $\mathrm{CSF}, 9 \%$ for GM and 7\% for WM. And for largest RF value, DPSOFCM-3 performs better than FCM-3: $4 \%$ for CSF, $8 \%$ for GM and 6\% for WM. From this, we can infer that our improved algorithm is less sensitive to Noise and RF than FCM-3

To further determine the quality and correctness of our method, we consider performance in terms of the widely used multi-class metrics: as True Positive (TP), as False Negative (FN) and as False Positive (FP) (Tables 4 and 5).

Table 4. Overall Classification table for FCM-3: 0\% Noise and 0\% RF (a); 9\% Noise and 40\% RF (b).

\begin{tabular}{|c|c|c|c|c|c|c|}
\hline & \multicolumn{2}{|c|}{ TP } & \multicolumn{2}{c|}{ FN } & \multicolumn{2}{c|}{ FP } \\
\hline & (a) & (b) & (a) & (b) & (a) & (b) \\
\hline CSF & 97.69 & 93.69 & 2.31 & 6.31 & 4.81 & 7.97 \\
\hline GM & 95.91 & 82.64 & 4.09 & 17.36 & 1.30 & 9.98 \\
\hline WM & 99.33 & 90.77 & 0.67 & 9.23 & 2.97 & 17.13 \\
\hline
\end{tabular}

Table 5. Overall Classification table for DPSOFCM-3: 0\% Noise and $0 \%$ RF (a); $9 \%$ Noise and $40 \%$ RF (b).

\begin{tabular}{|c|c|c|c|c|c|c|}
\hline & \multicolumn{2}{|c|}{ TP } & \multicolumn{2}{c|}{ FN } & \multicolumn{2}{c|}{ FP } \\
\hline & (a) & (b) & (a) & (b) & (a) & (b) \\
\hline CSF & 97.80 & 95.12 & 2.20 & 4.88 & 3.71 & 5.26 \\
\hline GM & 96.71 & 91.77 & 3.29 & 8.23 & 0.90 & 3.08 \\
\hline WM & 99.63 & 97.12 & 0.37 & 2.88 & 2.03 & 7.88 \\
\hline
\end{tabular}

On Table 4, we compare FCM-3 metrics between extreme cases: (0\% Noise, $0 \%$ RF) against (9\% Noise, $40 \% \mathrm{RF}$ ). The drawback from defects is obvious as TP metric is lower for case (b) than case (a), while FN and FP metrics are much higher.
On Table 5, we compare DPSOFCM-3 metrics between the same extreme cases, and the improvement is quite significant. Indeed, the metric differences have decreased which indicates a lower sensitivity to noise and RF.

In order to better exploit these metrics, we can also measure performance by using Precision and Sensitivity defined as follow:

$$
\begin{aligned}
\text { Precision } & =\frac{T P}{T P+F P} \\
\text { Sensitivity } & =\frac{T P}{T P+F N}
\end{aligned}
$$

Table 6. Comparison of Precision $(P)$-sensitivity $(S)$ between FCM3 and DPSOFCM-3. (a): 0\% Noise and 0\% RF; (b): 9\% Noise and $40 \%$ RF.

\begin{tabular}{|c|c|c|c|c|}
\hline & \multicolumn{5}{|c|}{ FCM-3 } \\
\hline & \multicolumn{2}{|c|}{$P \times 100$} & (a) & (b) \\
\hline & (a) & (b) & 98 & 94 \\
\hline CSF & 95 & 92 & 96 & 83 \\
\hline GM & 99 & 89 & 96 & 91 \\
\hline WM & 97 & 84 & 99 & $($ DPSOFCM-3 \\
\hline & \multicolumn{5}{|c|}{ DP } & \multicolumn{3}{c|}{$S \times 100$} \\
\hline & (a) & (b) & (a) & 96 \\
\hline & 96 & 96 & 96 & 99 \\
\hline CSF & 99 & 99 & 99 & 98 \\
\hline GM & 98 & 98 & 98 &
\end{tabular}

On Table 6, we can clearly see that FCM-3 looses precision and sensitivity with noisy and non-uniform images, while DPSOFCM-3 is much more stable.

\section{Conclusions and Future Work}

In this article, we have presented results of segmentation of brain MRI three-variate images using FCM-3. The results obtained by using FCM-3 algorithm show that the drawback of such FCM type algorithms still persists but is somewhat reduced when considering the three modalities T1, T2, and PD.

As we have seen, these results were greatly improved by as much as $9 \%$ by the use of the extension to multivariate modalities to DPSO prior to FCM. This hybridation which we have named DPSOFCM-3 is very promising and can be improved further either by iterating the whole process or by incorporating spatial neighbourhood information [26] extended to multivariate modalities.

So far, we have only checked performance with respect to ground truth and not speed performance. For future work, we are working on parallel version of our method to speed up performance on dedicated high performance computers $[2,16]$.

The parameters we have used in this study need further investigation. Apart from the number of classes $c=4$ and the fuzziness $m=2$, the other parameters where found in the literature and are also the object of future work. 


\section{References}

[1] Agrawal S., Panda R., and Dora L., "A Study on Fuzzy Clustering for Magnetic Resonance Brain Image Segmentation Using Soft Computing Approaches," Applied Soft Computing, vol. 24, pp. 522-533, 2014.

[2] Al-Adwan A., Sharieh A., and Mahafzah B., "Parallel Heuristic Local Search Algorithm on OTIS Hyper Hexa-Cell and OTIS Mesh of Trees Optoelectronic Architectures," Applied Intelligence, vol. 49, no. 2, pp. 661-688, 2019.

[3] Al-Shaikh A., Mahafzah B., and Al Sharaideh M., "Metaheuristic Approach Using Grey Wolf Optimizer for Finding Strongly Connected Components in Digraphs," Journal of Theoretical and Applied Information Technology, vol. 97, no. 16, pp. 4439-4452, 2019.

[4] Bezdek J., Pattern Recognition with Fuzzy Objective Function Algorithms, Advanced Applications in Pattern Recognitio, 1981.

[5] Cocosco C., Kollokian V., Kwan R., and Evans A., "BrainWeb: Online Interface to a 3D MRI Simulated Brain Database," NeuroImage, vol. 5, no. 4, pp. 425, 1997.

[6] Dora L., Agrawal S., Panda R., and Abraham A., "State of the Art Methods for Brain Tissue Segmentation: A Review," IEEE Reviews in Biomedical Engineering, vol. 10, pp. 235-249, 2017.

[7] Dubey Y. and Mushrif M., "FCM Clustering Algorithms for Segmentation of Brain MR Images," Advances in Fuzzy Systems, pp. 1-14, 2016.

[8] Dunn J., "A Fuzzy Relative of The Isodata Process and Its use in Detecting Compact Well Separated Clusters," Journal of Cybernetics, vol. 3, no. 3, pp. 32-57, 1973.

[9] Filho T., Pimentel B., Souza R., and Oliveira A., "Hybrid Methods for Fuzzy Clustering Based on Fuzzy C-Means and Improved Particle Swarm Optimization," Expert Systems with Applications, vol. 42, no. 17, pp. 6315-6328, 2015.

[10] Jain A., Murty M., and Flynn P., "Data Clustering: A Review," ACM Computing Surveys, vol. 31, no. 3, pp. 264-323, 1999.

[11] Kannan S., Ramathlagam S., Devi R., and Hines E., "Strong Fuzzy C-Means in Medical Image Data Analysis," Journal of Systems and Software, vol. 85, no. 11, pp. 2425-2438, 2012.

[12] Kathiravan S. and Kanakaraj J., "A Review on Potential Issues and Challenges in MR Imaging," The Scientific World Journal, pp. 1-10, 2013.

[13] Kennedy J. and Eberhart R., "Particle Swarm Optimization," in Proceedings of ICNN'95International Conference on Neural Networks, Australia, Perth, pp. 1942-1948, 1995.
[14] Khattab H., Sharieh A., Mahafzah B., "Most Valuable Player Algorithm for Solving Minimum Vertex Cover Problem," International Journal of Advanced Computer Science and Applications, vol. 10, no. 8, pp. 159-167, 2019.

[15] Li H., He H., and Wen Y., "Dynamic Particle Swarm Optimization and K-means Clustering Algorithm for Image Segmentation," OptikInternational Journal for Light and Electron Optics, vol. 126, no. 24, pp. 4817-4822, 2015.

[16] Mahafzah B., "Performance Evaluation of Parallel Multithreaded A* Heuristic Search Algorithm," Journal of Information Science, vol. 40, no. 3, pp. 363-375, 2014.

[17] Masadeh R., Mahafzah B., and Sharieh A., "Sea Lion Optimization Algorithm," International Journal of Advanced Computer Science and Applications, vol. 10, no. 5, pp. 388-395, 2019.

[18] Masadeh R., Sharieh A., and Mahafzah B., "Humpback Whale Optimization Algorithm Based on Vocal Behavior for Task Scheduling In Cloud Computing," International Journal of Advanced Science and Technology, vol. 13, no. 3, pp. 121-140, 2019.

[19] Masulli F., Schenone A., and Massone A., Fuzzy Systems in Medicine, Springer-Verlag Berlin Heidelberg, 2000.

[20] Mekhmoukh A. and Mokrani K., "MR Brain Image Segmentation Using an Improved Kernel Fuzzy Local Information C-Means Based Wavelet, Particle Swarm Optimization Initialization and Outlier Rejection with Level Set Methods," The International Arab Journal of Information Technology, vol. 15, no. 4, pp. 683692, 2018.

[21] Mirghasemi S., Rayudu R., and Zhang M., “A New Modification of Fuzzy C-Means via Particle Swarm Optimization for Noisy Image Segmentation," in Proceedings of Australasian Conference on Artificial Life and Computational Intelligence, Canberra, pp. 147-159, 2016.

[22] Nayak J., Naik B., and Behara H., Computational Intelligence in Data Mining-Volume 2, Spring Link, 2015.

[23] Saneipour K. and Mohammad M., "Improvement of MRI Brain Image Segmentation Using Fuzzy Unsupervised Learning," Iranian Journal of Radiology, vol. 16, no. 2, pp. 1-6, 2019.

[24] Saxenal N., Tripathi A., Mishra K., and Misra A., "Dynamic-PSO: An Improved Particle Swarm Optimizer," in Proceedings of IEEE Congress on Evolutionary Computation, Sendai, pp. 212-219, 2015.

[25] Semchedine M. and Moussaoui A., "An Effcient Particle Swarm Optimization for MRI Fuzzy Segmentation," Romanian Journal of 
Information Science and Technology, vol. 20, no. 3, pp. 271-285, 2017.

[26] Song J. and Zhang Z., "Brain Tissue Segmentation and Bias Field Correction of MR Image Based on Spatially Coherent FCM with Nonlocal Constraints," Computational and Mathematical Methods in Medicine, vol. 3, pp. 113, 2019.

[27] Tavares J., "Image Processing and Analysis: Applications and Trends," in Proceedings of, AES-ATEMA $5^{\text {th }}$ International Conference, on Advances and Trends in Engineering Materials and their Applications, Montreal and Quebec City, Canada, pp. 27-42, 2010.

[28] Venkatesan A. and Parthiban L., "Medical Image Segmentation with Fuzzy C-Means and Kernelized Fuzzy C-Means Hybridized on PSO and QPSO," The International Arab Journal of Information Technology, vol. 14, no. 1, pp. 53-59 2017.

[29] Zanaty E., "Determination of Gray Matter (GM) and White Matter (WM) Volume in Brain Magnetic Resonance Images (MRI)," International Journal of Computer Applications, vol. 45, no. 3, pp.16-22, 2012.

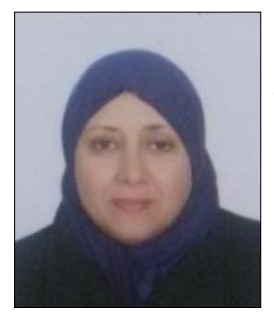

Kies Karima is currently an assistant professor and a permanent member of SIMPA laboratory in informatics department at University of Science and Technology of Oran-Mohamed Boudiaf (USTO-MB). She received her engineering degree in Computer Science, M.Sc. and Ph.D from USTO-MB (19992009). She is the head of Computer Science department and has published more than ten papers in journals and conference proceedings. Her main research interests include medical image processing, $3 \mathrm{D}$ image segmentation and pattern recognition.

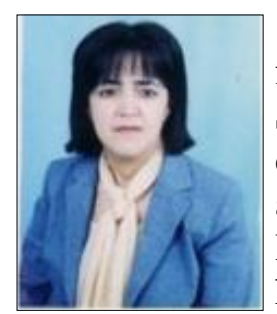

Benamrane Nacera is currently a full professor and a director of SIMPA laboratory in informatics department at University of Science and Technology of Oran-Mohamed Boudiaf (USTO-MB). She received her engineering degree in Computer Science from University of Oran, the M.Sc. and Ph.D. degrees from University of Valenciennes, France, in 1988 and 1994. Since 2002, she is the head of vision and medical imaging team at SIMPA laboratory. She has published more than 90 papers in journals and conference proceedings. Her main research interests include image processing, medical imaging, computer vision, biomedical engineering and pattern recognition. 